\title{
The way ahead for fusion
}

\author{
As the construction of the ITER tokamak enters its next phase - the machine assembly - now is a good time for a \\ recap of the history and current status of nuclear fusion research.
}

S ometimes small changes can have a big effect. When Ernest Rutherford, Marcus Oliphant and Paul Harteck carried out experiments in 1934, they were "surprised to find that on bombarding heavy hydrogen with diplons [deuterons] an enormous effect was produced"1, whereas in previous studies with alpha particles impinging on a deuterium target, no significant effect had been observed. Besides the discovery of tritium and helium-3, their experiment achieved the first fusion reactions in the laboratory - fusion research took off with a bang.

Among the first concepts of magnetic confinement fusion devices were the stellarator (with its helically twisted plasma shape), proposed by the American physicist Lyman Spitzer, and the tokamak (which confines the plasma in the form of a donut), introduced by Soviet scientists Andrei Sakharov and Igor Tamm. Another approach (inertial confinement fusion), put forward by John Nuckolls and colleagues, implodes capsules filled with heavy hydrogen isotopes using high-energy laser beams ${ }^{2}$.

Today's fusion community actively explores all these paths - alongside other concepts - as we highlighted in a special Insight issue back in $2016^{3}$. In September 2019, the Nature Conference "Advances and Applications in Plasma Physics" not only brought together scientists working on different fusion concepts but also connected the wider plasma physics community - from astrophysical plasmas to laser-plasma interactions, cold/dusty plasmas and applications. In the sessions dedicated to magnetic confinement fusion, the ambivalence of the audience was tangible: will the ITER tokamak (which is Latin for 'the way') live up to expectations and demonstrate the feasibility of nuclear fusion power?

Large-scale international projects come into existence on the timescale of decades rather than years, and ITER is no exception. As Matteo Barbarino recounts in a Comment on the history of nuclear fusion, the ITER collaboration was founded more than thirty years ago by the European Union (through the Euratom treaty), Japan, the Soviet Union and the United States, who were later joined by the People's Republic of China, the Republic of Korea and India. From the initial proposal to the start of

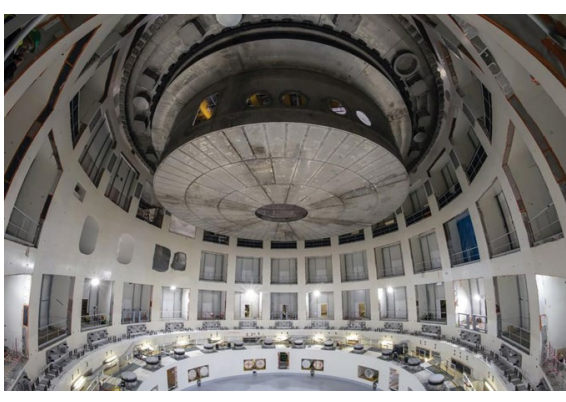

Credit: ITER Organization

construction in the South of France, around twenty years should pass.

Problems with the design and management issues delayed the start of operations and led to a substantially revised budget of roughly $€ 20$ billion. However, following the arrival of Director-General Bernard Bigot in 2015 , a root and branch review of the project led to a new baseline design. This revised schedule marked a turning point for the construction of ITER: the first hydrogen plasma discharge is expected in 2025, and the work to achieve this is $70 \%$ complete and therefore on track. We spoke to Bernard Bigot and Tim Luce, head of ITER's Science \& Operations Department, about the status of the project. You can read the conversation elsewhere in this issue.

The progress is most strikingly visible at the ITER worksite in St. Paul-lez-Durance, France. Earlier this year, the tokamak building was handed over to the ITER Organization and the 1,250-tonne cryostat base was installed within the required precision of only $3 \mathrm{~mm}$. With the first major components, such as toroidal or poloidal magnetic field coils, also arriving on site, the focus has now shifted from the construction of the infrastructure to machine assembly, a milestone that was celebrated on 28 July.

The next milestones include the commissioning of the liquid helium cryogenic plant and the pre-assembly of the (nine) vacuum vessel sectors with two toroidal magnetic field coils each and thermal shielding, with the first module expected to be installed towards the end of the year. In addition, the first of six magnets making up the central solenoid is expected to be shipped from the United States to France later this year. The solenoid will be the last major component to be installed before the cryostat will be closed up in late 2024 , followed by a period of integrated commissioning and testing to prepare for First Plasma.

Of course, these milestones might require some adjustment due to the ongoing COVID-19 pandemic: work on site has been reduced to critical activities with precautionary safety measures in place and lockdown measures such as the temporary closures of workshops or factories in individual member states might affect the delivery of critical components. A report on the impact is expected this month, but Bernard Bigot believes this not to be a major setback.

The strong performance and vigorous pace of progress demonstrated by the ITER Organization in reaching critical milestones of the new baseline has also shifted public perception. The conversation is now less cynical compared to the first, rather troubled, years of the project - with the growing public awareness that the issue of climate change becomes ever more urgent. After First Plasma, installation of critical components for deuterium-tritium operation such as additional heating or a fuel recycling system will prepare the machine for its ultimate goal: the demonstration of the feasibility of fusion power is expected to commence in 2035. This seems like a long way down the road, but ITER's recent track record is impressive.

Although ITER will not be the immediate solution to the problem of clean and affordable energy, it will be crucial in informing the design of the next generation of fusion reactors that will produce electricity instead of heat. Scientists are thinking way ahead: conceptual designs for commercial tokamaks as a globally distributable, sustainable supply of energy are already underway. This is the way towards a future with safe and sustainable energy.

Published online: 28 August 2020 https://doi.org/10.1038/s41567-020-01043-9

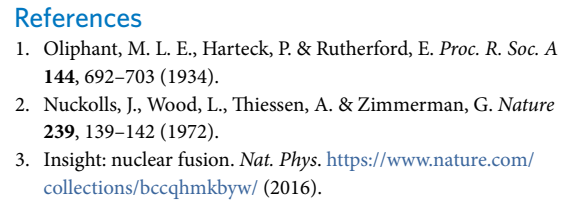

